\title{
Energy-Efficient Transmission for Beamforming in Wireless Sensor Networks
}

\author{
Jing Feng, Che-Wei Chang, Serkan Sayilir, Yung-Hsiang Lu, Byunghoo Jung, \\ Dimitrios Peroulis, and Y. Charlie Hu \\ School of Electrical and Computer Engineering, Purdue University, West Lafayette, Indiana 47906, USA
}

\begin{abstract}
Energy conservation is essential in wireless sensor networks (WSNs) because of limited energy in nodes' batteries. Collaborative beamforming uses multiple transmitters to form antenna arrays; the electromagnetic waves from these antenna arrays can create constructive interferences at the receiver and increase the transmission distance. Each transmitter can use lower power and save energy, since the energy consumption is spread over multiple transmitters. However, if the same nodes are always used, these nodes would deplete their energy much sooner and this sensing area will no longer be monitored. To avoid this situation, energy consumption for collaborative beamforming needs to be balanced over the whole network by assigning the transmitters in turns. The transmitters in each round are selected by a scheduler and the energy carried in each node is balanced to increase the number of transmissions. The lifetime of a network is the number of transmissions until a certain percentage of the nodes depletes their energy. This paper proposes an algorithm to calculate energy-efficient schedules based on the remaining energy and the phase differences of their signals arriving at the receiver. Compared with an existing algorithm, our algorithm can extend the network lifetime by more than $60 \%$.
\end{abstract}

\section{INTRODUCTION}

Wireless sensor networks (WSNs) have attracted researchers' attention due to their broad applications. Energy conservation is essential in WSNs because nodes are usually battery-powered. In WSNs, wireless communication for long distance is a major consumer of energy [4]. Recent studies suggest that cooperative communication (CC) [8], [10], [15] and collaborative beamforming [9], [11], [12] can save transmission energy. Among CC techniques, multipleinput/multiple-output (MIMO) uses multiple antennae at both the transmitter and the receiver to improve performance. However, nodes are usually small and each node can have only one antenna; multiple antennae may be too costly for each node. Therefore, traditional MIMO cannot be directly applied to wireless sensor networks. Miller et al. [10] propose a new technique in CC: the receiver detects the signal based on the distortion of the radiation pattern due to multiple paths. Their method does not improve signal strength in the direction of the receiver. In contrast, this paper uses beamforming to increase signal strength in the intended direction. Collaborative beamforming can increase signal gain in a particular direction by using antenna arrays to form constructive electromagnetic waves. This can be useful in some applications where the distance between two hops is too far by using a single transmitter. Collaborative beamforming also enhances the energy efficiency of the system. Compared with direct transmission from one transmitter to the receiver or hop-by-hop transmission, beamforming spreads the energy consumption over multiple transmitters and improves the signal strength at the receiver [4]. Therefore, individual transmitters can use less energy for the same receiver. This balances the energy consumption over the entire network and extends the network lifetime. Beamforming may be used in applications such as (1) ecology monitoring in a forest when nodes are deployed far away from the receiver in a laboratory, (2) information from ground sensors sent to a satellite, and (3) secure data dissemination, when signals cannot be detected in unintended directions.

Beamforming efficiency [7] depends on the phase differences between the electromagnetic waves that arrive at the receiver. It is $100 \%$ when phase differences are zero. Assuming each transmitter has the same transmitted power and free-space attenuation, for $N$ transmitters, the receiving signal has $N^{2}$ power gain. It can increase the transmission range by $N$ times farther. Alternatively each transmitter can reduce its power to $1 / N^{2}$ for the same distance. However, in practice, phase differences may occur from several sources, such as frequency offsets, transmitters' locations, and initial phase offsets. The phase differences between two nodes can be estimated by using two-way signal exchanges [14]. Beamforming efficiency is higher when choosing the transmitters with smaller phase differences [5]. For better performance, the nodes with small phase differences are always used and these nodes will deplete their energy much faster than the other nodes. This may cause coverage holes: areas not monitored because sensor nodes run out of energy. To prevent coverage holes, energy consumption should be balanced among the nodes.

In this paper, we propose an algorithm to schedule transmitting nodes for collaborative beamforming. We define a network's lifetime as the number of successful beamforming transmissions before a percentage of nodes are energyexhausted. Our scheduling algorithm selects the transmitters in each round from $N$ nodes within a circle of radius $\rho$. Figure 1 shows an example where the nodes need to transmit data to a receiver at point $D$. Because the distance from the sensing area to the receiver is large, the signal transmitted by an individual node cannot reach the receiver. Therefore, the nodes form antenna arrays to create directional radio waves with higher signal strength in the direction of the receiver. The signal strength must be $G_{m}(\mathrm{~dB})$ times greater than the signal from a single transmitter. In this figure, four sensor nodes are selected to transmit. To schedule the transmitters for beamforming transmissions while prolonging network lifetime, our algorithm is designed based on three rules: (i) The remaining energy in all nodes needs to be balanced. (ii) The signal gain at the receiver exceeds the minimum level. (iii) The amount of data transmitted to the receiver is maximized. 


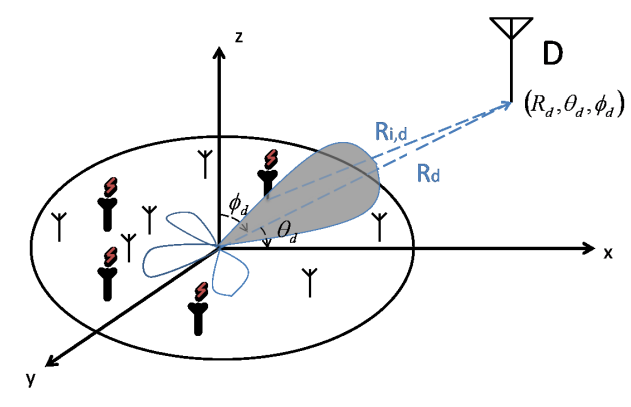

Fig. 1. Distributed beamforming. Ten nodes are randomly deployed in a circle. The shaded area shows the radiated wave pattern with the selected four transmitters (four antennae in bold).

To our knowledge, this is the first paper that focuses on energy-efficient scheduling for collaborative beamforming. We propose an algorithm to schedule the participating nodes in each round of transmission. Our simulation results show that our method can extend the network lifetime by more than $60 \%$ compared with an existing transmission scheduling algorithm [5]. We also validate that beamforming can increase the signal strength in a particular direction through an outdoor experiment.

\section{RELATED WORK}

\section{A. Beamforming}

Collaborative beamforming is a transmission technique by using antenna arrays to achieve directional signal transmission or reception [6]. Recent studies show that collaborative beamforming can improve energy efficiency for wireless sensor networks [2], [12], [16], [17]. Ochiai et al. [12] analyze the characteristics of collaborative beamforming's patterns when transmitting sensor nodes are uniformly deployed in a circle. Ahmed and Vorobyov [2] show that collaborative beamforming provides better performance when nodes are deployed with Gaussian distribution. Zarifi et al. [17] show that when the nodes are uniformly deployed in a circle, selecting transmitting nodes from a ring of proper radii can narrow the main lobe of the average beam pattern. All these studies show collaborative beamforming can increase the signal strength at the intended direction and extend the transmission range. However, electromagnetic waves from different nodes may arrive at the receiver with different phases, due to various reasons, such as different distances and initial phase offsets. Methods proposed in [2], [12], [17] assume that the selected nodes can adjust their phases to cancel the phase differences.

\section{B. Phase Partition}

Traditional beamforming algorithms require the transmitters to adjust the phases, so that the signals received at the destination are constructive. Chang et al. [5] propose phase partition (PP): instead of controlling the phases, PP divides transmitters into several groups based on their phases. Each round of beamforming uses only one group. PP does not consider the remaining energy in each node and the signal strength at the receiver; the method is not energy-efficient in achieving long network lifetime in three cases: (i) Too many transmitters are assigned to the same group and the received signal strength is much higher than necessary. (ii)
TABLE I

Simulation PARAMETERS

\begin{tabular}{|l|c|l|}
\hline Parameter & Symbol & Value \\
Packet Size & $b$ & $180 \mathrm{bits}$ \\
Packet Rate & $\mathrm{PR}$ & 1 packet/sec \\
Free Space path-loss exponent & $\beta$ & 2 \\
Energy consumed on radio circuitry & $\epsilon_{e}$ & $50 \mathrm{~nJ} / \mathrm{bit}$ \\
Energy consumed on radio amplifier & $\epsilon_{a}$ & $100 \mathrm{pJ} / \mathrm{bit}$ \\
Transmission distance & $\mathrm{d}$ & meter \\
Node Energy (maximum) & $E_{\max }$ & $1000 \mathrm{~J}$ \\
\hline
\end{tabular}

The transmitters in the same group have different amounts of energy, if they are deployed at different times. When the group is selected to transmit, the nodes with less energy will deplete their energy sooner and create coverage holes. (iii) The transmitters are assigned into groups based on their phases. As a result, the numbers of nodes in different groups can be different. In Section IV-A, we introduce an improved phase partition method (IPP) with multiple transmitting power levels by reducing the excessive signal strength at the receiver.

\section{Energy Model}

We use the energy model presented in [13]. $E_{t x}(b, d)$ is the total energy consumption for one node transmitting $b$ bits of data to the distance $d ; E_{r x}(b)$ is the energy consumption for one node receiving $b$ bits of data. Table I defines the symbols and lists the values used in this paper.

$$
\begin{aligned}
& E_{t x}(b, d)=\epsilon_{e} \times b+\epsilon_{a} \times b \times d^{\beta} \\
& E_{r x}(b)=\epsilon_{e} \times b
\end{aligned}
$$

\section{Contributions}

This paper has the following contributions: (1) We propose an algorithm, energy and phase (EP), to schedule the transmitting nodes based on their remaining energy and relative phase differences at the receiver in order to prolong the network lifetime. Both PP and EP achieve beamforming transmissions without adjusting each node's phase. EP is different from PP in the following ways: (i) By considering the remaining energy in each node, EP can substantially extend the network lifetime. (ii) EP reduces the over-transmitted power by selecting the nodes based on the minimum signal strength for meeting the receiver's requirement. (2) We introduce an improvement on the existing algorithm, improved phase partition (IPP), by considering the minimum required signal strength, $G_{m}$, at the receiver. IPP allows the transmitters to adjust their transmission power and reduces the over-transmitted power. Our simulation results show that EP still achieves more transmissions compared with IPP. (3) This is the first paper that proposes a method to schedule transmitting nodes for beamforming while prolonging the network lifetime.

\section{ENERGy-EFFICIENT SCHEdULING Algorithm}

In this section, we focus on transmission scheduling to balance the energy consumption while prolonging the network lifetime. We show that optimal scheduling has a complexity that increases exponentially as the number of nodes increases. Hence, we propose a heuristic algorithm to schedule beamforming transmission while balancing the energy consumption over the entire network. 


\section{A. Problem Description}

$N$ nodes are deployed within a circle of radius $\rho$ from the origin in a polar coordinate system. The nodes are located in the horizontal plane, zenith angle $\theta_{i}=\pi / 2$ for $i=1, \ldots, N$, shown in Fig 1. Each node has an initial phase offset $\varphi_{i}$, carries energy $E_{i}$, and is located at $\left(R_{i}, \theta_{i}=\pi / 2, \phi_{i}\right) . E_{i} \leq E_{\max }$, where $E_{\max }$ is the maximum energy contained in one node. $R_{i}$ is the distance from the $i^{t h}$ node to the origin; $\theta_{i} \in[0, \pi]$ and $\phi_{i} \in[-\pi, \pi]$ are the zenith and azimuth angles [3]. The receiver is located at $\left(R_{d}, \theta_{d}, \phi_{d}\right)$. The nodes are synchronized and deployed in clusters, and the receiver is far away from these nodes, i.e. $R_{d} \gg \rho$. Hence, we assume that the propagation loss is the same for all transmitters. All the assumptions are also used in [12]. Each node has maximum transmission power $P_{\max }$ and the signal strength at the receiver needs to be $G_{m}$ times greater than the signal from a single transmitter. All antennae are omni-directional and the initial phase offsets, carried energy, and locations are known by a control center, and the transmission schedule can be computed offline. Then the transmission schedule is broadcasted to all nodes. All transmitters have the same information before sending it to the receiver. This may be achieved by using existing protocols, for example [15]. We define one transmission as a group of selected nodes using collaborative beamforming to transmit one packet to the receiver. Maximizing the total amount of data transmitted is equivalent to maximizing the number of rounds. The network lifetime, $p$, is the number of transmissions received by the receiver before $\eta \%$ nodes are energy-exhausted. Table II lists the symbols used in this paper. For simplicity, this paper does not consider near-field interference or multiple-path propagation.

The phase difference of the electromagnetic waves from each node at the receiver, $\Delta \varphi_{i}\left(\theta_{d}, \phi_{d}\right)$, can be calculated as

$$
\Delta \varphi_{i}\left(\theta_{d}, \phi_{d}\right)=\varphi_{i}+\frac{2 \pi}{\lambda} R_{i, d}
$$

$\Delta \varphi_{i}\left(\theta_{d}, \phi_{d}\right) \in[-\pi, \pi] . R_{i, d}$ is the distance between the $i^{t h}$ node and the receiver.

$$
\begin{array}{ll}
R_{i, d} & =\sqrt{\left(X_{i}-X_{d}\right)^{2}+\left(Y_{i}-Y_{d}\right)^{2}+\left(Z_{i}-Z_{d}\right)^{2}} \\
\text { where } & X_{i}=R_{i} \cos \phi_{i}, X_{d}=R_{d} \sin \theta_{d} \cos \phi_{d} \\
& Y_{i}=R_{i} \sin \phi_{i}, Y_{d}=R_{d} \sin \theta_{d} \sin \phi_{d} \\
& Z_{i}=0, Z_{d}=R_{d} \cos \theta_{d} .
\end{array}
$$

For each round of beamforming transmission, the signal strength at the receiver, $r(t)$, is:

$$
r(t)=\Re\left(\sum_{i=1}^{N} \alpha_{i k} \cdot e^{j\left(2 \pi f t+\Delta \varphi_{i}\right)}\right),
$$

here $\Re$ is the operator taking the real part; we assume that each node has only one power level to transmit. The term $\alpha_{i k}$ is the transmission coefficient: $\alpha_{i k}=1$ when the $i^{\text {th }}$ node transmits in the $k^{\text {th }}$ round and zero otherwise.

To extend the network lifetime while balancing the energy consumption, we need to satisfy the following conditions: (i) The beam formed by the signals has the main lobe pointing to the receiver. (ii) If the $i^{\text {th }}$ node transmits $p$ times (i.e.

\begin{tabular}{|c|c|c|c|}
\hline Symbol & Section & Definition & Unit \\
\hline $\bar{N}$ & $\mathrm{I}$ & Number of Nodes & \\
\hline$\rho$ & I & Deployment Radius & $\mathrm{m}$ \\
\hline$G_{m}$ & I & Minimum Signal Gain at Receiver & $\mathrm{dB}$ \\
\hline$E_{t x}(b, d)$ & II-C & $\begin{array}{l}\text { Energy consumption for one node to } \\
\text { transmit } b \text { bits data to distance } d\end{array}$ & $\mathrm{~J}$ \\
\hline$E_{r x}(b)$ & II-C & $\begin{array}{l}\text { Energy consumption for one node to } \\
\text { receive } b \text { bits }\end{array}$ & $\mathrm{J}$ \\
\hline$E_{\max }$ & II-C & $\begin{array}{l}\text { Maximum Energy Contained in One } \\
\text { Node }\end{array}$ & $\mathrm{J}$ \\
\hline$E_{i}$ & III-A & Initial Energy in Node $i$ & $\mathrm{~J}$ \\
\hline$E_{c, i k}$ & III-A & $\begin{array}{l}\text { Energy Consumed on Node } i \text { in round } \\
k\end{array}$ & $\mathrm{~J}$ \\
\hline$\epsilon_{e}$ & II-C & Energy consumed on radio circuitry & $\mathrm{J}$ \\
\hline$\epsilon_{a}$ & II-C & Energy consumed on power amplifier & $\mathrm{J}$ \\
\hline$\beta$ & II-C & Path-loss Exponent & \\
\hline$G_{a}$ & III-A & Gain of Antenna Arrays & $\mathrm{dB}$ \\
\hline$p$ & III-A & Network Lifetime & \\
\hline$R_{i}$ & III-A & Distance from Node $i$ to Origin & $\mathrm{m}$ \\
\hline$R_{d}$ & III-A & Distance from Receiver to Origin & $\mathrm{m}$ \\
\hline$R_{i, d}$ & III-A & Distance from Node $i$ to Receiver & $\mathrm{m}$ \\
\hline$\theta_{i}$ & III-A & Zenith Angle of Node $i$ & $\mathrm{rad}$ \\
\hline$\phi_{i}$ & III-A & Azimuth Angle of Node $i$ & $\mathrm{rad}$ \\
\hline$\theta_{d}$ & III-A & Zenith Angle for the Receive & $\mathrm{rad}$ \\
\hline$\phi_{d}$ & III-A & Azimuth Angle for the Receiver & $\mathrm{rad}$ \\
\hline$\varphi_{i}$ & III-A & Initial Phase Offset of Node $i$ & $\operatorname{rad}$ \\
\hline$\Delta \varphi_{i}$ & III-A & $\begin{array}{l}\text { Phase Difference of Signal from Node } \\
i \text {, at Receiver }\end{array}$ & $\mathrm{rad}$ \\
\hline$\alpha_{i k}$ & III-A & $\begin{array}{l}\text { Transmission Coefficient for Node } i \text { in } \\
\text { round } k\end{array}$ & \\
\hline$\eta$ & III-A & $\begin{array}{l}\text { Percentage of Energy Exhausted Nodes } \\
(0 \leq \eta \leq 100)\end{array}$ & $\%$ \\
\hline$U$ & III-A & Radiation Intensity & $\begin{array}{l}\text { W/unit } \\
\text { angle }\end{array}$ \\
\hline$A F$ & III-A & Array Factor & \\
\hline$P_{\max }$ & III-A & $\begin{array}{l}\text { Maximum Transmitting Power of One } \\
\text { Node }\end{array}$ & $\mathrm{mW}$ \\
\hline$\gamma$ & III-C & Rotating Degree & rad \\
\hline$P P$ & {$[5]$} & Phase Partition Method & \\
\hline$I P P$ & IV-A & Improved Phase Partition Method & \\
\hline$E P$ & III-C & Energy and Phase, Our Method & \\
\hline
\end{tabular}
the network lifetime), the total energy consumption does not exceed $E_{i}$, the initial energy carried by the node. (iii) Using beamforming, the gain of the constructive signals at the
TABLE II

SYMBOLS USED IN THIS PAPER

receiver is $G_{a}$ and $G_{a} \geq G_{m}$. The wasted power is minimized: $G_{a}-G_{m}$ is positive but as close to zero as possible. (iv) The energy consumption is balanced among all the nodes. To determine whether these conditions are met, we need to know the radiation intensity (signal strength) of the constructive electromagnetic waves in each direction.

For a group of $N$ transmitters, the radiation intensity at a far-field point [3] is expressed as:

$$
U(\theta, \phi)=U_{0}(\theta, \phi)|A F(\theta, \phi)|^{2},
$$

where $U_{0}$ is the antenna element factor and the beamforming array factor $A F$ is

$$
A F(\theta, \phi)=\sum_{i=1}^{N} e^{j\left(\Delta \varphi_{i}(\theta, \phi)\right)},
$$

$\Delta \varphi_{i}(\theta, \phi)$ is the phase difference of the signal from the $i^{t h}$ transmitter at the far-field point $\left(R_{d}, \theta, \phi\right)$ and can be calculated using equation (2). In $k^{t h}$ beamforming transmission $(k=1, \ldots, p)$, the signal gain $G_{a}$ at the receiver's location $\left(R_{d}, \theta_{d}, \phi_{d}\right)$ can be expressed as:

$$
G_{a}\left(\theta_{d}, \phi_{d}\right)=\left|A F\left(\theta_{d}, \phi_{d}\right)\right|^{2}=\left|\sum_{i=1}^{N} \alpha_{i k} \cdot e^{j\left(\Delta \varphi_{i}\left(\theta_{d}, \phi_{d}\right)\right)}\right|^{2}
$$




\section{B. Optimal Scheduling for Beamforming Transmissions}

Goal: Find a schedule $\alpha$ to balance the energy consumption over the network and maximize $p$. Here $\alpha$ is an $N \times p$ matrix, $\alpha_{i k}$ is either 0 or 1 with $i$ representing the $i^{t h}$ node, $i=1, \ldots, N$, and $k$ indicates the current round of transmission, $k=1, \ldots, p$. The network lifetime $p$ is the number of transmissions achieved before $\eta \%$ nodes are energy-exhausted. Four conditions listed in Section III-A can be formulated as:

\section{Constraints:}

1) The maximum radiation intensity is at the receiver's direction. Directivity of an antenna array is defined as the ratio of the radiation intensity from the antenna array in a given direction to the radiation intensity averaged over all directions [3]. Directivity $D_{k}\left(\theta_{d}, \phi_{d}\right)$ needs to be the maximum, i.e. the main lobe points to the receiver.

$$
\begin{aligned}
D_{k}\left(\theta_{d}, \phi_{d}\right) & =\max \left(D_{k}(\theta, \phi)\right) \\
& =\max \left(\frac{4 \pi \cdot U_{k}\left(\theta_{d}, \phi_{d}\right)}{\int_{0}^{2 \pi} \int_{0}^{\pi \pi} U_{k}(\theta, \phi) \sin \theta d \theta d \phi}\right) .
\end{aligned}
$$

$$
\text { where } U_{k}(\theta, \phi)=U_{0}(\theta, \phi)\left|\sum_{i=1}^{N} \alpha_{i k} \cdot e^{j\left(\Delta \varphi_{i}(\theta, \phi)\right)}\right|^{2}
$$

2) Total energy consumed on each transmitter is no greater than its initial energy.

$$
\sum_{k=1}^{p} \alpha_{i k} \cdot E_{c, i k} \leq E_{i}, \forall i, 1 \leq i \leq N .
$$

3) For each round, the signal strength at the receiver, $G_{a}\left(\theta_{d}, \phi_{d}\right)$ is at least $G_{m}$ times stronger than the signal transmitted by a single transmitter.

$$
G_{a}\left(\theta_{d}, \phi_{d}\right) \geq G_{m}, \forall k, 1 \leq k \leq p .
$$

4) After $k$ rounds of transmissions, no more than $\eta \%$ of nodes are energy exhausted, i.e. $1-\eta \%$ of nodes have remaining energy for at least one more transmission.

$$
\sum_{i=1}^{N} \sigma\left(E_{i}-E_{c, i k}-\sum_{k=1}^{p} \alpha_{i k} \cdot E_{c, i k}\right)>(1-\eta \%) \cdot N,
$$

where $\sigma(v)$ is the sign function:

$$
\sigma(v)= \begin{cases}1, & v \geq 0 \\ 0, & v<0 .\end{cases}
$$

Transmission coefficient $\alpha_{i k}$ is required to be 0 or 1 for all $i=1, \ldots, N$ and $k=1, \ldots, p$. There are $2^{N p}$ potential schedules for $N$ nodes with $p$ rounds of transmissions. To verify each potential schedule with the four constraints, the complexity is $O(N p)$. Hence the total computation complexity to find the optimal schedule $\alpha$ is $O\left(2^{N p}\right)$. In this paper, we propose a heuristic method for scheduling. We design an algorithm which schedules nodes in each transmission based on their remaining energy and phase differences at the receiver.

\section{Scheduling Algorithm Based on Energy and Phase (EP)}

A desirable algorithm should achieve more transmissions before the same number of nodes become energy-exhausted. To prevent the nodes with low remaining energy from being energy-exhausted, the nodes with higher remaining energy should be selected first. Meanwhile, the phase differences of transmitters are also important. Two signals are canceled

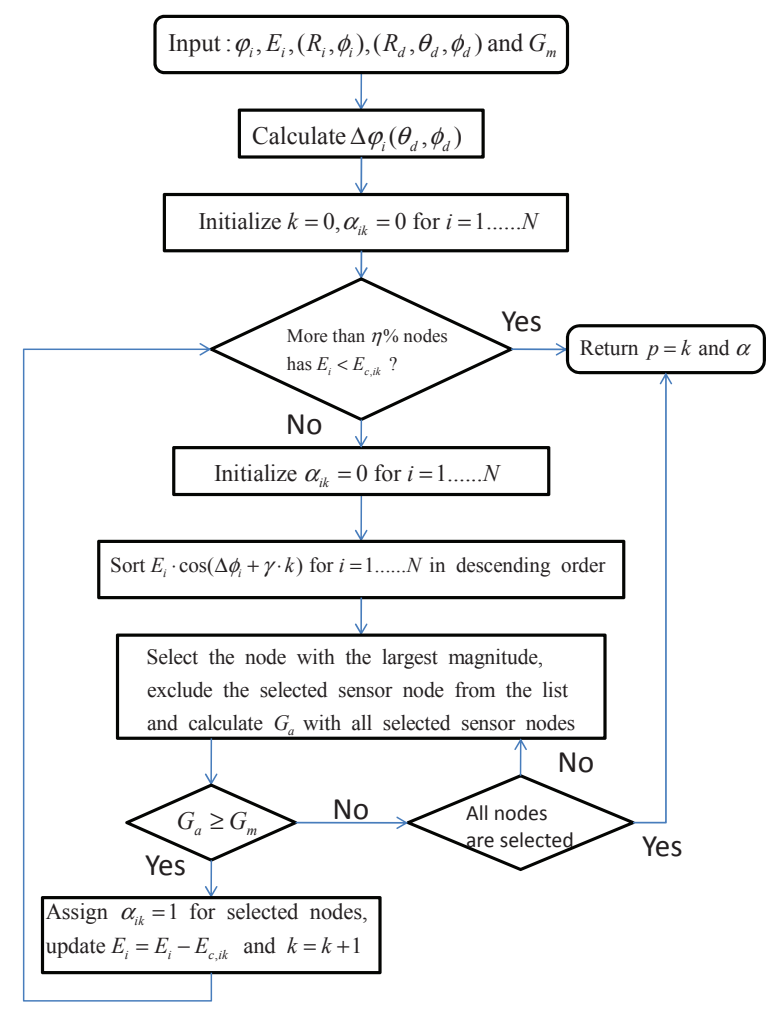

Fig. 2. Flowchart of Energy and Phase method

when they have 180 degrees phase difference. Therefore, the nodes participating in each transmission cannot be chosen based only on their remaining energy. Equation (4) shows that smaller phase differences provide a larger gain. Our algorithm chooses the nodes to participate in each transmission by giving higher priorities to the nodes with (i) more remaining energy, (ii) smaller phase differences relative to a reference phase at the receiver. Equation (10) expresses the relationship between the selected nodes and the corresponding signal gain at the receiver in the $k^{t h}$ transmission from Equation (4):

$$
\begin{aligned}
& G_{a}\left(\theta_{d}, \phi_{d}\right) \\
& =\left|\sum_{i=1}^{N} \alpha_{i k} \cos \left(\Delta \varphi_{i}\left(\theta_{d}, \phi_{d}\right)\right)+j \sum_{i=1}^{N} \alpha_{i k} \sin \left(\Delta \varphi_{i}\left(\theta_{d}, \phi_{d}\right)\right)\right|^{2} \\
& =\sum_{i=1}^{N} \sum_{h=1}^{N} \alpha_{i k} \cdot \alpha_{h k} \cdot \cos \left(\Delta \varphi_{i}\left(\theta_{d}, \phi_{d}\right)-\Delta \varphi_{h}\left(\theta_{d}, \phi_{d}\right)\right) .
\end{aligned}
$$

Here $\alpha_{i k}$ and $\alpha_{h k}(1 \leq i, h \leq N, 1 \leq k \leq p)$ are the transmission coefficients and $\left(\Delta \varphi_{i}\left(\theta_{d}, \phi_{d}\right)-\Delta \varphi_{h}\left(\theta_{d}, \phi_{d}\right)\right) \in$ $[-\pi, \pi]$. Equation (10) shows that the gain of the antenna array is related to the phase differences among the selected transmitters.

For each round $k$, we sort the nodes with their products of remaining energy $E_{i}$ and $\cos \left(\Delta \varphi_{i}+\gamma \cdot k\right)$ in descending order to determine their priorities. Here $\Delta \varphi_{i}$ is the phase difference of node $i, \gamma \cdot k$ is the reference phase at the receiver, and we name $\gamma$ to be the rotating degree. Rotating degree $\gamma$ is used to avoid the case that some of the nodes can never be selected. Since $E_{i}$ is always a non-negative number and $\cos \Delta \varphi_{i}$ is in the range of [-1,1], the product $E_{i} \cdot \cos \Delta \varphi_{i}$ is non-negative when $-\pi / 2 \leq \Delta \varphi_{i} \leq \pi / 2$. As a result, a transmitter is never selected when $\Delta \varphi_{i}$ is outside the range $[-\pi / 2, \pi / 2]$. However, 
$G_{a}$ is determined by the relative phase differences between selected transmitters, not the absolute values of phases. To avoid repetitively using the same nodes for transmissions, we rotate the reference phase by $\gamma$ degrees after each transmission. The value of $\gamma$ has little effect on the overall beamforming as shown in Section IV-C1.

To minimize the wasted power, we select nodes one by one based on their priorities, the products $E_{i} \cdot \cos \left(\Delta \varphi_{i}+\gamma \cdot k\right)$. After one node is selected, we calculate $G_{a}$ with all currently selected nodes. If $G_{a} \geq G_{m}$, currently selected nodes are assigned to transmit for this round. These selected nodes have their transmission coefficient $\alpha_{i k}=1$. We increase $k$ by 1 and recompute the number of energy-exhausted nodes. If this number is smaller than $\eta \%$, we calculate the priorities with their updated remaining energy and select the nodes for the next round of transmission. If the number of energy exhausted nodes is equal to or larger than $\eta \%$, we stop and return the current value of $k$ as the network lifetime. The scheduling algorithm terminates if $G_{a}<G_{m}$ even when all nodes with positive remaining energy are selected. This means no more beamforming transmission can be achieved even though some nodes still have energy left. Their phase differences are too big to create a strong enough signal in the direction of the receiver. Figure 2 shows the flowchart of EP.

For one round of transmission, the complexity to calculate the product for priorities is $O(N)$, to sort the priorities into descending order requires $O(N \log N)$. To calculate gain after one transmitter is added requires time complexity $O(N)$ using equation (4). Since the maximum number of nodes that can be selected in each round is $N$ and there are $p$ rounds of transmissions, the gain calculation has a complexity of $O\left(N^{2} p\right)$. This occurs only when all nodes are required to be selected. In the worst case, the complexity of our algorithm is $O\left(N^{2} p\right)$. The complexity can be reduced in two ways. (1) From $G_{m}$, at least $10^{\frac{G_{m}}{20}}\left(G_{m}\right.$ is the power gain in $\mathrm{dB}$ and with no phase difference, $N$ transmitters can achieve $N^{2}$ signal gain in the intended direction) transmitters are needed to make $G_{a}=G_{m}$; hence, EP can start with $10^{\frac{G_{m}}{20}}$ transmitters. (2) Instead of adding one transmitter each time, multiple transmitters can be selected so that $G_{a}$ approaches $G_{m}$ faster.

\section{Simulation, ANALYsis, AND EXPERIMENTS}

Since PP does not adjust the number of transmitters based on the signal strength required by the receiver, all nodes in the same group transmit using their highest power in each round. When the number of nodes is more than necessary, the network lifetime is shorter because their transmitted power is too high. Different from PP, EP schedules the transmitters based on the minimum required signal gain at the receiver. To make a fair comparison, we introduce an improved PP that reduces the signal strength at the receiver by using lower transmitting power.

\section{A. Improved PP with Multiple Power Levels (IPP)}

In this section, we improve phase partition by assuming each node can use a lower transmitting power to avoid the overtransmitted power at the receiver. The network lifetime using this improved phase partition method (IPP) is then compared
TABLE III

SCENARIOS CONSIDERED IN THIS PAPER AND THE RATIO OF $p$ USING EP, IPP, AND PP TO THE UPPER BOUND. UPPER BOUND FOR A1-A3 AND C1-C3 IS 6250; FOR B1-B3 AND D1-D3 Is 3125.

\begin{tabular}{|c|c|r|l|c|c|}
\hline Cases & Algorithm & Radius $\rho$ & $E_{i}(\mathrm{~J})$ & $\begin{array}{c}\text { p for } \\
\eta=100\end{array}$ & Ratio \\
\hline A1 & EP & $100 \mathrm{~m}$ & $E_{\max }$ & 5592 & $89 \%$ \\
A2 & IPP & $100 \mathrm{~m}$ & $E_{\max }$ & 5530 & $88 \%$ \\
A3 & PP & $100 \mathrm{~m}$ & $E_{\max }$ & 3438 & $55 \%$ \\
B1 & EP & $100 \mathrm{~m}$ & $\left(0, E_{\max }\right]$ & 2807 & $90 \%$ \\
B2 & IPP & $100 \mathrm{~m}$ & $\left(0, E_{\max }\right]$ & 1863 & $60 \%$ \\
B3 & PP & $100 \mathrm{~m}$ & $\left(0, E_{\max }\right]$ & 1368 & $44 \%$ \\
C1 & EP & $1000 \mathrm{~m}$ & $E_{\max }$ & 5571 & $89 \%$ \\
C2 & IPP & $1000 \mathrm{~m}$ & $E_{\max }$ & 5540 & $89 \%$ \\
C3 & PP & $1000 \mathrm{~m}$ & $E_{\max }$ & 3500 & $56 \%$ \\
D1 & EP & $1000 \mathrm{~m}$ & $\left(0, E_{\max }\right]$ & 2814 & $90 \%$ \\
D2 & IPP & $1000 \mathrm{~m}$ & $\left(0, E_{\max }\right]$ & 1853 & $60 \%$ \\
D3 & PP & $1000 \mathrm{~m}$ & $\left(0, E_{\max }\right]$ & 1356 & $43 \%$ \\
\hline
\end{tabular}

with PP and EP. By allowing multiple transmitting power levels, the normalized transmitting power (i.e. transmission coefficient, $\alpha_{i k}$ ) of each transmitter is a discrete number in range [0,1]. CC2420 RF transceiver [1] is commonly used in MICAz and other popular nodes and CC2420 offers 31 discrete RF power levels. Hence, we let $\alpha_{i k}$ be discrete in $[0,1]$ with evenly divided 31 levels. Improved PP (IPP) calculates the total phase difference of the transmitted signal from each node at the receiver and then groups nodes based on their phase differences. IPP estimates the signal strength at the receiver based on the number of nodes that still have energy for transmissions in the currently selected group and adjust the transmitters' power. For example, if all nodes transmit using $P_{\max }$, the signal strength at the receiver is 4 times higher than $G_{m}$, then each node reduces its transmission power level to $\frac{P_{\max }}{4}$. The over transmitted power at the receiver can be reduced and the energy is saved for later transmissions; therefore, network lifetime is extended. In this paper, to reduce the algorithm's complexity, we assume that using IPP, the transmission coefficients for the nodes in the same group are adjusted to the same level, even though the remaining energy of each node in the same group may not be the same.

\section{B. Evaluations}

For $N=100$ nodes, we consider 12 scenarios with 3 factors: (1) scheduling algorithm, EP, PP, or IPP with 31 power levels; (2) deployment radius $\rho, 100$ meters or 1000 meters; (3) distribution of initial energy $E_{i}, E_{i}=E_{\max }$ for all nodes or $E_{i}$ is uniformly random distributed in range $\left(0, E_{\max }\right]$. All scenarios are listed in Table III. The receiver is at a far-field point on the x-axis, $R_{d}=10000 \mathrm{~m}, \theta_{d}=\pi / 2, \phi_{d}=0$. From Section II-C, $E_{\max }=1000 \mathrm{~J}$ and the energy consumption $E_{c, i k}=E_{t x}\left(b, R_{i, d}\right)$, where $b$ is the size of the transmitting data in bits, $R_{i, d}$ is the distance from the node $i$ to the receiver. The receiver requires the minimum signal gain, $G_{m}$, to be $20 \mathrm{~dB}$, i.e. the gain of radiated power at the receiver to be 100 times higher than using an individual transmitter. Since the radiation power is proportional to the square of the amplitude of the radio wave, $G_{m}=20 \mathrm{~dB}$ requires 10 transmitters if they have no phase difference. In all scenarios, $R_{d}=10000 \mathrm{~m}$, hence, the upper bound of $p$ for $N=100$ nodes is $\frac{E_{\max } \cdot N}{10 \cdot E_{t x}\left(b, R_{d}\right)}=6250$ when $E_{i}=E_{\max }$. In other scenarios, when $E_{i}$ is uniform random distributed between $(0$, $\left.E_{\max }\right]$, the expected mean value of $E_{i}$ is $\frac{E_{\max }}{2}$ and the upper 


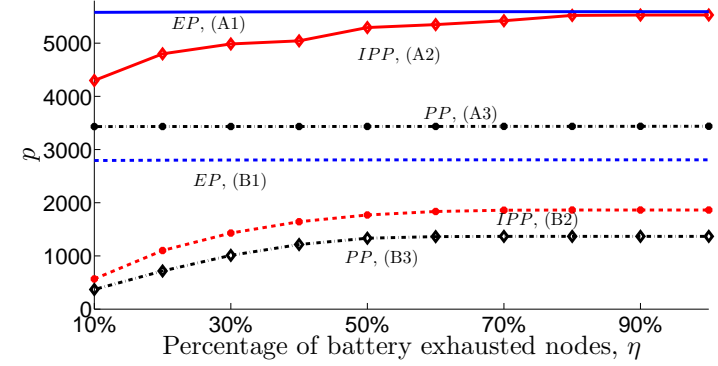

(a) $N=100, \rho=100 \mathrm{~m}$

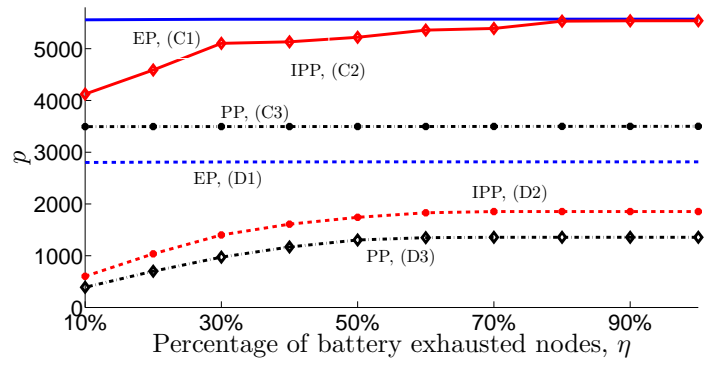

(b) $N=100, \rho=1000 \mathrm{~m}$

Fig. 3. Network lifetime, $p$, increases as higher percentages of energy exhausted nodes, $\eta$.

bound of $p$ is 3125 . We use $\gamma=2 \pi / 6$ in EP, 6 groups in $\mathrm{PP}$ and IPP in comparisons. From the simulations, Table III lists the network lifetime at $\eta \%=100 \%$ in all scenarios and the ratio of the network lifetime using EP, IPP, and PP to the upper bound of $p$. The simulation results show that in all scenarios, EP achieves nearly $90 \%$ network lifetime compared with the upper bound. For A1, A3, C1, and C3, EP extends the lifetime by almost $60 \%$. For scenarios B1, B3, D1, and D3, EP almost doubles the network lifetime. EP achieves slightly more transmissions (roughly 1\%) than IPP in scenarios A1$\mathrm{A} 2$ and $\mathrm{C} 1-\mathrm{C} 2$; in scenarios B1-B2 and D1-D2, EP achieves approximately $50 \%$ more transmissions respecting to IPP. We further evaluate EP by comparing it with IPP and PP with the following metrics: (i) network lifetime with different numbers of energy-exhausted nodes, (ii) signal strength at the receiver in each round, (iii) remaining energy after 1000 rounds of transmissions, (iv) directivity, e.g. round $k=500,1000$, and 2000.

1) Network Lifetime: Figures 3(a) and 3(b) show the number of successful beamforming transmissions (i.e. $G_{a} \geq G_{m}$ ) versus the percentage of battery-exhausted nodes. A better algorithm can provide a longer network lifetime for a given $\eta$. In Figure 3(a), the six lines from the top to the bottom show the network lifetime $p$ with $\eta$ changes in scenarios $\mathrm{A} 1-\mathrm{A} 3$ and B1-B3. Figure 3(b) shows the scenarios C1-C3 and D1-D3. From these figures we see that EP extends the network lifetime by at least $60 \%$ compared with PP. This is because phase partition divides transmitters into groups without considering their remaining energy. Transmitters in the same group participate in the same number of transmissions. However, their initial energy may be different, in scenarios B3 and D3. Since nodes in the same group carry different amounts of initial energy, after some rounds of transmission, nodes with low initial energy become energy-exhausted. In B3 and D3, the number of energy-exhausted transmitters grows as the number of transmissions increases for $\eta \% \leq 50 \%$. When $\eta \%>50 \%, p$ saturates. This is because after these many transmissions, too many nodes are energy-exhausted in some groups and the signals from these groups are too weak for the receiver. When this happens, $p$ becomes a constant as $\eta$ increases. When the initially carried energy for all nodes is the same, this problem doesn't occur. As shown in scenarios $\mathrm{A} 3$ and $\mathrm{C} 3, E_{i}=1000 \mathrm{~J}$, using PP makes all sensor nodes deplete their energy almost at the same time. However, if the number of nodes in a group is more than necessary, the signal strength at the receiver is higher than the requirement and energy is wasted. The network lifetime can be further extended by reducing this over-transmitted power.

Compared with PP, IPP reduces the over-transmitted power. With a small enough step-size (e.g. 1/31 in these simulations), the gain of the signal at the receiver can be adjusted to meet the required signal gain. In Figure 3(a) and 3(b), IPP prolongs the network lifetime by $60 \%$ and $58 \%$ in scenarios A2 and C2. In scenarios B2 and D2, using IPP, nodes in the same group may not have the same amount of initial energy, but the same number of transmissions are assigned to these nodes. As $k$ increases, nodes with low initial energy become energy-exhausted. This causes the number of available nodes in some groups to become too small to achieve beamforming transmissions and reach the receiver, even though some nodes still have large amounts of remaining energy. Therefore, IPP cannot extend network lifetime too much when the initial energy in each node is random.

Different from PP and IPP, EP gives higher priorities to the nodes with more remaining energy and the energy consumption is balanced among all nodes. Therefore, regardless of the condition of initial carried energy, all nodes start to deplete their energy at approximately the same time. In scenarios B1 and D1, the initial energy in all nodes is between 0 and $1000 \mathrm{~J}$; in scenarios $\mathrm{A} 1$ and $\mathrm{C} 1, E_{i}=1000 \mathrm{~J}$ for all nodes. EP schedules transmissions to the nodes with higher remaining energy. As a result, the network lifetime is almost a constant as the number of energy-exhausted nodes increases. Compared to PP, EP (scenario A1 and C1) has larger $p$ 's because EP reduces the over-transmitted power by using only the necessary number of transmitters. Since IPP reduces the transmitting power, it achieves the same network lifetime as EP. We do not consider propagation loss and energy consumed on data sharing, so $\rho$ has little effect on network lifetimes. In the following analysis, we use scenarios B1-B3 to analyze the performance of EP, PP and IPP.

2) $G_{a}$ in Each Transmission: The receiver requires a minimum signal gain, $G_{m}$. When the signal gain at the receiver is much larger than $G_{m}$, energy is wasted. Figure 4 shows that using EP, the gain of the antenna arrays at the receiver is very close to $G_{m}$ in all rounds. $G_{m}$ is satisfied for all rounds of transmissions, until no more beamforming transmission can be achieved. In contrast, PP exceeds $G_{m}$ substantially in the first 1000 rounds of transmissions and wastes energy. After 1000 rounds of transmissions, the numbers of remaining nodes in some groups are too small; hence the signal transmitted by these groups cannot be received. Compared with PP, in the 


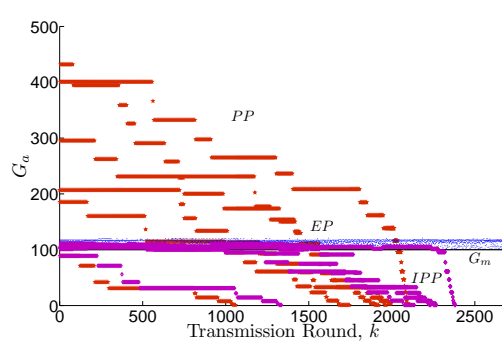

Fig. 4. Gain of antenna array in each round of beamforming transmission (Scenarios B1-B3).

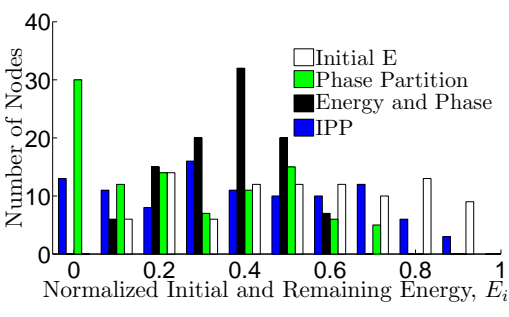

Fig. 5. Distribution of $E_{i}$ before and after 1000 rounds of beamforming transmission using PP, EP, and IPP (Scenarios B1-B3).

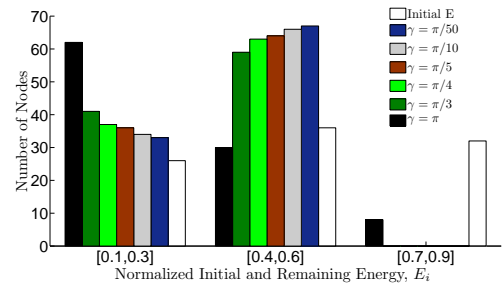

Fig. 6. Energy distribution before and after 1000 rounds of transmissions with different $\gamma$ in scenario B1. first few hundreds rounds of transmission, IPP reduces the signal gain to meet $G_{m}$. The energy saved in these rounds of transmissions are used in the later transmissions. Hence, in the later transmissions, IPP has stronger signal gain than PP.

3) Change in Remaining Energy: Considering scenarios B1-B3 in Table III, Figures 5 shows the distribution of the initial energy and the remaining energy after 1000 rounds of beamforming transmissions using EP, IPP, and PP. Using $\mathrm{EP}$, no node depletes its energy. Most of the nodes have remaining energy greater than $30 \%$ and the number of nodes with low energy are kept almost the same as in initial carried energy. Compared with both IPP and PP, EP has fewer energyexhausted nodes for the same number of transmissions and prolongs the network lifetime.

4) Directivity: Figures 7(a)-7(c) show the radiation patterns in the $\mathrm{x}$-y plane, where $\theta=\pi / 2, \phi=[0,2 \pi]$, using both methods in the transmission rounds $k=500,1000$, and 2000 . In each figure, the solid line represents the radiation pattern when EP is used; the dashed line shows the radiation pattern when PP is used; the dash-dotted line is the radiation pattern when IPP with 31 transmission power levels is used. As mentioned in Section III-A, the main lobe should point to the direction of the receiver. The receiver is at $\left(R_{d}=10000 \mathrm{~m}\right.$, $\theta_{d}=\pi / 2, \phi_{d}=0$ ) in these simulations, so the main lobe should point to $0^{\circ}$. As expected, the main lobe in each figure points to the intended direction. The value of directivity in each plot shows the ratio of the radiation intensity in the intended direction to the radiation intensity averaged over all directions. In each figure, the radiation intensity in each angle is normalized to its local maximum value, i.e. the maximum radiation intensity in the 2-D plane when using all three algorithms in that round of transmission. Therefore, the radiation intensity in two different plots may not be normalized to the same value.

Since EP selects transmitters based on the required signal strength at the receiver, the radiation intensity of the solid line at $0^{\circ}$ is very close to the minimum radiation intensity required by the receiver. In the first few hundreds rounds of transmissions (e.g. 500, and 1000), phase partition wastes power by using too many transmitters. Figure 7(a), and 7(b) show that in the intended direction, PP has a larger gain compared with EP. As more transmissions have completed, some of the nodes deplete their energy. Thus the number of nodes in some groups decreases and their constructive electromagnetic waves are too weak to be received. Figure 7 (c) show that when $k=2000$, using phase partition, the signal cannot reach $G_{m}$ in the intended direction.
IPP adjusts transmission power to a lower level if the gain of the signal at the receiver is stronger than necessary. In Figure $7(a)-7(c)$, the shape of the IPP radiation patterns are the same as PP. In Figure 7(a) and 7(b), IPP has weaker signals than PP, i.e. IPP has less over-transmitted power than PP.

\section{Sensitivity Analysis of EP}

In this section we perform a sensitivity analysis on EP using the following metrics: (i) rotating degree of the reference phase $\gamma$, (ii) network lifetime affected by phase uncertainties.

1) Rotating Degree $\gamma$ : In Section III-C, we mentioned that in order to avoid using the same nodes repetitively, we rotate the phase reference at the receiver by $\gamma$ after each round of transmission. Since cosine is a periodic function for every $2 \pi$ and symmetric within $2 \pi$, we select $\gamma$ in the range of $(0, \pi]$. Figure 6 shows the energy distribution for initial and remaining energy after 1000 rounds of transmissions when $\gamma=\pi / 50, \pi / 10, \pi / 5, \pi / 4, \pi / 3$ and $\pi$. The initial energy is almost uniformly distributed. When $\gamma$ is too large (e.g. $\pi$ ), after 1000 rounds of transmissions, more than 60 nodes have energy less than $30 \%$ but nearly 10 nodes still have energy as high as $90 \%$. This shows that $\gamma$ cannot be $\pi$ to balance nodes' remaining energy. For $\gamma=\pi / 50, \pi / 10, \pi / 5, \pi / 4$, and $\pi / 3$, after 1000 rounds of transmissions, nearly 60 sensor nodes have $40 \%$ to $60 \%$ energy left and the number of nodes with low energy (i.e. less than $30 \%$ ) is around 40 . From the simulation, we see that the value of $\gamma$ has little effect on the energy distribution after 1000 rounds of transmissions as long as $\gamma$ is small.

2) Phase Uncertainty: So far, we assume that we know the location and the initial phase offset of each node. Therefore, we can calculate the phase difference of the signal from each transmitter. In this section, we relax the assumption and examine the effects when the phase difference of each node is uncertain. Our algorithm selects nodes for each round of transmission based on the estimated signal strength at the receiver. This requires accurate knowledge of phase differences. When the phase differences are uncertain, the actual transmitted signal strength may be different from the estimation and beamforming may fail. To compensate the uncertainties, we adjust our estimation based on beamforming efficiency. Beamforming efficiency $e$, is an estimated ratio of the achieved signal strength and the highest possible signal strength when the phase uncertainties of every node are in a certain range [7]. Figure 8(a) shows both amplitude and power when phases are uncertain. At point $\mathrm{A}$, the maximum phase uncertainty is $\pi / 3$ (i.e. all 100 nodes have phase uncertainties 


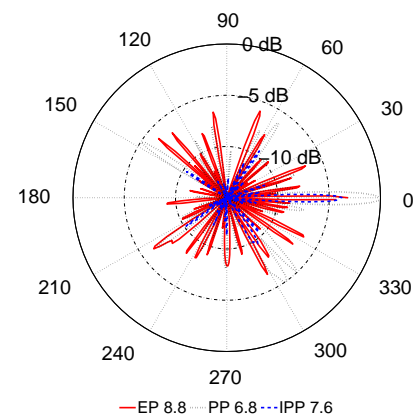

(a) $k=500$

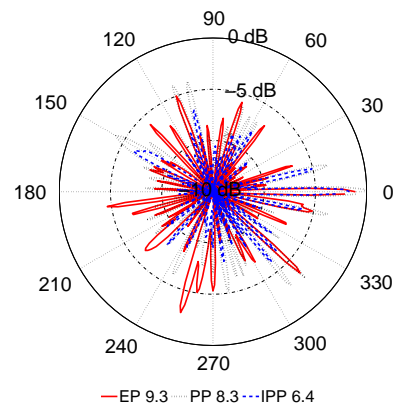

(b) $k=1000$

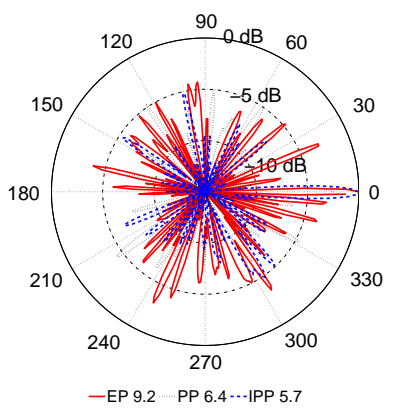

(c) $k=2000$

Fig. 7. Radiation patterns for the $k^{t h}$ round of transmission using EP, PP, and IPP. Legends on the bottom show the directivity. Since the selected transmitters in each round are different using EP, PP, and IPP, the directivity are different in each round. Compared with PP and IPP, EP always has a higher directivity, which means the radiating energy is more concentrated in the receiver's direction.

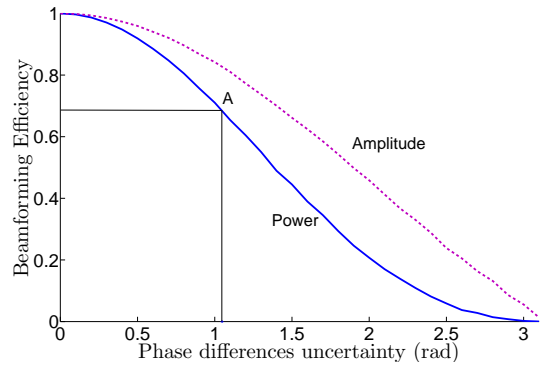

(a)

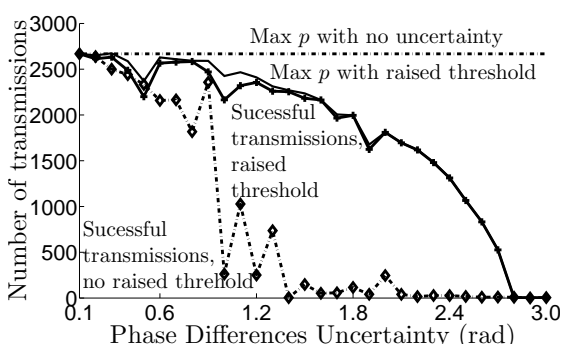

(b)

Fig. 8. (a) Beamforming efficiency vs phase uncertainty. For 100 nodes, phase uncertainties are randomly generated each time. Beamforming efficiency is averaged by 100 trials. (b) Using scenario B1 as an example, with the raised threshold, more transmissions become successful. (The curves have fluctuations due to the inputs are uniform random numbers.)

in the range of $[0, \pi / 3])$. The actual transmitted signal strength at this point is roughly $67 \%$ compared with the signal strength when the phases' have no uncertainty.

In Figure 8(b), the first line from the top shows the maximum number of transmissions achievable when there is no phase uncertainty. The number of successful transmissions is a constant. The lowest line is the actual number of transmissions that can be successfully received when the estimated phase is uncertain. As the phase uncertainty increases, the total number of successful transmissions decreases. For the phase uncertainty greater than $1.5 \mathrm{rad}$, there is almost no successful transmission. To compensate the phase uncertainties, we adjust our transmitter selecting threshold $G_{m}$ based on beamforming efficiency. Beamforming efficiency is an average signal strength for the given phase uncertainty, shown in Figure 8(a). According to Figure 8(a), we estimate that signal strength at the receiver would be $e \cdot G_{m}$, where $e$ is the corresponding beamforming efficiency for the given phase uncertainty. To compensate the phase uncertainties, we raise the threshold for each round of transmission from $G_{m}$ to $G_{m} / e$ and more nodes are selected in each transmission. The solid line shows the maximum number of transmissions that the network can achieve with the raised threshold and the number of transmissions decreases as the phase uncertainty increases. This is because the large phase uncertainty gives lower beamforming efficiency and requires a higher threshold. After applying this change to the threshold in EP, our simulation result shows that with the raised threshold, the number of successful transmissions increases compared with the lowest line. Since

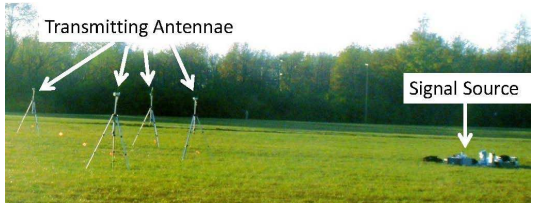

Fig. 9. Setup in our outdoor experiments.

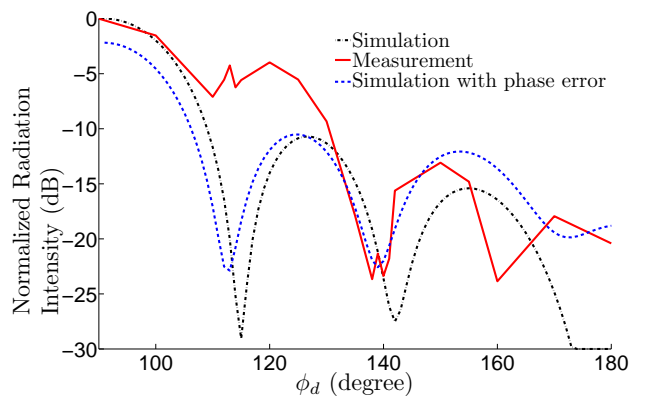

Fig. 11. Compare the radiation pattern at the angle from 90 to 180 degree.

phase uncertainty is random and $e$ represents the relationship between beamforming efficiency and phase uncertainty in the average case, the actual number of successful transmissions cannot reach the maximum number of transmissions shown by the solid line.

\section{Validation}

We designed a testbed and performed outdoor experiments with up to 8 transmitters, shown in Figure 9. The preliminary results roughly match the theoretical prediction of the radiation pattern and validate our simulation. These experiments were conducted in an empty soccer field. We use $27 \mathrm{MHz}$ as the carrier frequency $(\lambda \approx 11 \mathrm{~m})$. All antennae are connected to the same signal source through grounded coaxial cables. This setup ensures that the signal into each cable is in phase and has the same frequency. By changing the relative locations of the antennae, we measured the radiation patterns and compared them with the simulation results. The received power was 


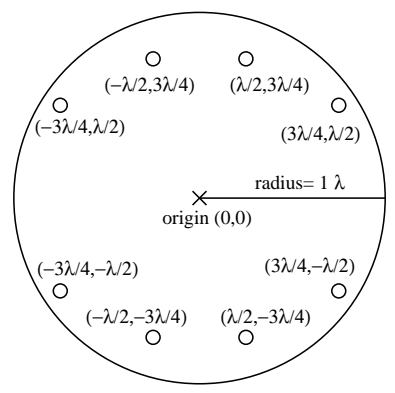

(a)

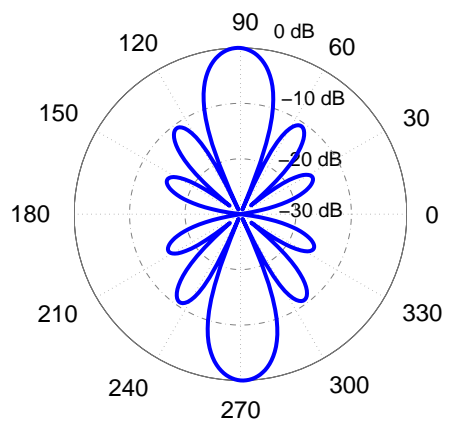

(b)

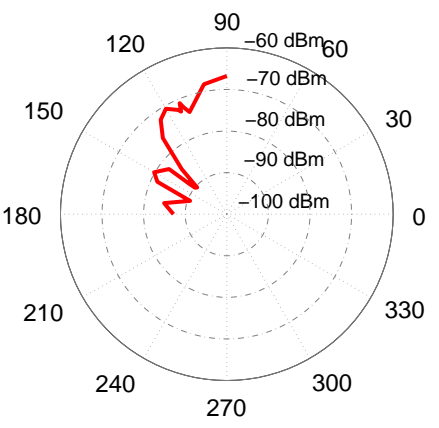

(c)

Fig. 10. (a) Eight transmitters deployed in a circle with radius $=1 \lambda$. (b) Simulation radiation pattern, signal strength in each angle is normalized to the maximum signal strength. (c) Radiation pattern from measurement, signal strength measured in $\mathrm{dBm}$.

measured and recorded by a power spectrum analyzer. The transmitters are approximately $90 \mathrm{~m}$ from the receiver.

Figure 10 shows one of the experiments with 8 transmitting antennae. Figure 10(b) shows the expected radiation pattern from simulation. Figure 10(c) shows the measured signal strength. Since the radiation pattern is symmetric, we measured the signal strength only in the second quadrant $\left(\phi_{d}=[\pi / 2, \pi]\right)$. In Figure 11, our data show similar radiation pattern as the simulation: has three peaks and three nulls, around $90^{\circ}, 125^{\circ}$, and $155^{\circ}$, with the highest peak point at $90^{\circ}$. The nulls are at around $115^{\circ}, 145^{\circ}$, and $170^{\circ}$. The radiation intensity at $90^{\circ}$ is about $25 \mathrm{~dB}$ stronger than at $140^{\circ}$. Our measured result does not show a null at $115^{\circ}$ and the pattern is shifted by roughly 10 degrees to the left. The error may arise due to the following reasons: (i) The locations of the transmitters and the receiver are not precise. We estimated that the maximum error in distance is $0.5 \mathrm{~m}$. (ii) The radiated signal from each transmitter has small phase difference. We measured the phase difference due to the cables and connectors and discovered that one pair had $30^{\circ}$ phase difference from the others. The other pairs had phase differences less than $3^{\circ}$. The dashed line in Figure 11 shows the simulation result when one of the transmitters has $30^{\circ}$ phase difference. This also shows a shift to the left, consistent with our measured result.

\section{CONCLUSION}

Collaborative beamforming increases the transmission range and enhances energy efficiency in wireless sensor systems. This is the first paper to study maximizing the network lifetime using collaborative beamforming. We propose a heuristic algorithm to schedule nodes for beamforming transmission. Our algorithm achieves $60 \%$ more beamforming transmissions than phase partition.

\section{FUTURE WORK}

Our work can be extended by considering multiple power levels in each transmitter and the energy consumed on data sharing. This paper assumes transmissions are ISI (intersymbol-interference) free, the highest transmitting powers and the energy loss due to propagation for all transmitters are the same. A more accurate energy model and a complex channel model (e.g. multi-path) may be adopted in a future study.

\section{ACKNOWLEDGMENTS}

This work is supported in part by CNS 0716271 and CNS 0509394. Any opinions, findings, and conclusions or recommendations in the project are those of the authors and do no necessarily reflect the views of the sponsor.

\section{REFERENCES}

[1] http://www-inst.eecs.berkeley.edu/ cs150/Documents/CC2420.pdf.

[2] M. F. A. Ahmed and S. A. Vorobyov. Collaborative beamforming for wireless sensor networks with gaussian distributed sensor nodes. IEEE Transactions on Wireless Communications, 8(2):638-643, Feb. 2009.

[3] C. A. Balanis. Antenna Theory: Analysis and Design. Wiley, 1997.

[4] S. M. Betz et al. Cooperative Beamforming and Power Control. In Asllomar Convference on Signals Systems and Computers,2007.

[5] C.-W. Chang et al. Radiating Sensor Selection for Distributed Beamforming in Wireless Sensor Networks. In Military Communications Conference, 2008.

[6] L. Dong et al. Cooperative Beamforming for Wireless Ad Hoc Networks. In IEEE Global Communications Coference, 2007.

[7] J. Feng, Y.-H. Lu, B. Jung, and D. Peroulis. Energy Efficient Collaborative Beamforming in Wireless Sensor Networks. In IEEE International Symposium on Circuits and Systems, 2009.

[8] S. Gupta et al. Power efficiency of cooperative communication in wireless sensor networks. In 3rd International Conference on Signal Processing and Communication Systems,pages 1-10,2009.

[9] S. Lakshmanan et al. Towards adaptive beamforming in indoor wireless networks: An experimental approach. In INFOCOM,2009.

[10] R. Miller et al. Radio Teaming: Establishing Communication when Communication is not Possible. In IEEE International Conference on Mobile Ad Hoc and Sensor Systems, pages 365-370,2008.

[11] R. Mudumbai, G. Barriac, and U. Madhow. On the Feasibility of Distributed Beamforming in Wireless Networks. IEEE Transactions on Wireless Communications, 6(5):1754-1763, May 2007.

[12] H. Ochiai, P. Mitran, H. V. Poor, and V. Tarokh. Collaborative Beamforming for Distributed Wireless Ad Hoc Sensor Networks. IEEE Transactions on Signal Processing, 53(11):4110-4124, November 2005.

[13] M. Perillo et al. An integrated approach to sensor role selection. IEEE Transactions on Mobile Computing, 8(5):709-720, May 2009.

[14] S. Sayilir et al. Phase difference and frequency offset estimation for collaborative beamforming in sensor networks. In IEEE International Symposium on Circuits and Systems, 2010.

[15] M. Z. Siam et al. Energy-efficient clustering/routing for cooperative mimo operation in sensor networks. In IEEE INFOCOM,2009.

[16] S. Tokunaga et al. Power saving effect of sensor collaborative beamforming for wireless ubiquitous network systems. In Asia-Pacific Conference on Communications, pages 1-5, 2008

[17] K. Zarifi et al. Distributed beamforming for wireless sensor networks with random node location. In IEEE International Conference on Acoustics, Speech and Signal Processing, pages 2261-2264,2009. 